Pure and Applied Mathematics Quarterly

Volume 5, Number 4

(Special Issue: In honor of

John Tate, Part 1 of 2)

$1435-1467,2009$

\title{
Half a Century of Rigid Analytic Spaces
}

\author{
Siegfried Bosch \\ Dedicated to John Tate on the occasion of his eightieth birthday.
}

\begin{abstract}
We explain the basic ideas and facts in rigid geometry from today's point of view. Starting out from Tate's Harvard notes, the classical approach by the school of Grauert and Remmert is covered, as well as the approach through formal schemes following Raynaud, including a glimpse on several advanced results.

Keywords: rigid analytic space, Tate elliptic curve, Tate's Acyclicity Theorem affinoid space, affinoid subdomain, Grothendieck topology, formal scheme, admissible blowing-up, flattening, Reduced Fiber Theorem.
\end{abstract}

After the $p$-adic numbers had been discovered by Hensel in 1893, there were several attempts to develop a theory of analytic functions over $p$-adic fields. At first one was just curious about knowing if there would exist a reasonable analogue of classical complex function theory over such fields. However, later when algebraic geometry had progressed so that applications to number theory were possible, a good theory of analytic functions, say over $\mathbb{C}_{p}$ (the completed algebraic closure of $\mathbb{Q}_{p}$ ), became sort of a necessity.

There is a fundamental example, due to J. Tate, which provided ample motivation for the development of such a new theory. Let $K$ be an algebraically closed field with a complete non-Archimedean absolute value $|\cdot|$, which is assumed to

Received November 30, 2006. 
be non-trivial in the sense that there are elements $a \in K$ with $|a| \neq 0,1$; for example, we may take $K=\mathbb{C}_{p}$. Then, using $\zeta$ as a variable, look at the algebra

$$
\mathcal{O}\left(K^{*}\right)=\left\{\sum_{\nu \in \mathbb{Z}} c_{\nu} \zeta^{\nu} ; c_{\nu} \in K, \lim _{|\nu| \rightarrow \infty}\left|c_{\nu}\right| r^{\nu}=0 \text { for all } r>0\right\}
$$

of all Laurent series that are globally convergent on $K^{*}$. Viewing $\mathcal{O}\left(K^{*}\right)$ as the ring of analytic functions on $K^{*}$, we can construct its field of fractions $\mathcal{M}\left(K^{*}\right)=$ $Q\left(\mathcal{O}\left(K^{*}\right)\right)$ and think of it as of the field of meromorphic functions on $K^{*}$.

Now choose an element $q \in K^{*}$ with $|q|<1$, and write $\mathcal{M}^{q}\left(K^{*}\right)$ for the set of all meromorphic functions which are invariant under multiplication by $q$ on $K^{*}$; i. e.,

$$
\mathcal{M}^{q}\left(K^{*}\right)=\left\{f \in \mathcal{M}\left(K^{*}\right) ; f(q \zeta)=f(\zeta)\right\}
$$

Tate made the observation that $\mathcal{M}^{q}\left(K^{*}\right)$ is an elliptic function field with a nonintegral $j$-invariant, i. e., with $|j|>1$. Furthermore, he saw that the set of $K$-valued points of the associated elliptic curve $E_{K}$ coincides canonically with the quotient $K^{*} / q^{\mathbb{Z}}$. Elliptic curves which are obtained in this way have been called Tate elliptic curves since then. As quotients of type $K^{*} / q^{\mathbb{Z}}$ are not meaningful in the setting of algebraic geometry, Tate was tempted to develop a theory of analytic spaces, so-called rigid analytic spaces, where such quotients make sense; see his notes on a seminar he gave at Harvard [T]. In fact, the existence of an analytical isomorphism of type $E_{K} \simeq K^{*} / q^{\mathbb{Z}}$ is a charcterizing condition for Tate elliptic curves.

One can prove that, just as in the classical complex case, isomorphism classes of elliptic curves correspond one-to-one to isomorphism classes of Riemann surfaces of genus 1 in the sense of rigid analytic spaces. Among these precisely the elliptic curves with non-integral $j$-invariant are Tate elliptic, whereas all others extend to elliptic curves over the valuation ring of $K$ and are said to have good reduction (assuming $K$ algebraically closed, as above). Tate elliptic curves may be viewed as the correct analogues of complex tori. However, they can only be viewed as multiplicative quotients of type $K^{*} / q^{\mathbb{Z}}$, since the additive point of view, as applied in the complex case, will not work. An obvious reason for this is that the exponential function, if defined at all, does not converge well enough. 


\section{An overview}

When they were distributed, Tate's fundamental notes on Rigid Analytic Spaces [T] immediately received strong attention for several reasons. First, there was the fascinating design of a new analytic theory which, for the first time, was able to manage, even in higher dimensions, the problem that fields with a nonArchimedean absolute value are totally disconnected and, thus, sheaves of locally analytic functions (in the usual sense) are much too big. Tate achieved this through his famous Acyclicity Theorem which, at the expense of replacing the topology on his spaces by a certain Grothendieck topology, allowed to define analytic functions in local terms. The extra constraints provided by the Grothendieck topology are reflected in the term rigid which Tate introduced in order to specify his notion of analytic spaces. On the other hand, Tate's notes contained several comments marked as "Open Questions", which served as convenient starting points for further research. Picking up such questions, the school of Grauert and Remmert, with their expertise in classical complex analysis, started introducing new methods into the theory; see the monography [BGR]. In particular, relying on techniques based on the Weierstraß Preparation Theorem, Gerritzen and Grauert [GG] were able to clarify the structure of affinoid subdomains, and to characterize open immersions of affinoid spaces as inclusions of subdomains. Thereby two major problems left open by Tate were settled. Subsequent work by Kiehl [K1], [K2] established Theorems A and B for coherent modules on rigid spaces, as well as the Proper Mapping Theorem. All this was ample evidence for the fact that Tate's original approach was sound and wisely chosen.

Another source of inspiration certainly has come from Grothendieck. Tate himself writes at the beginning of $[\mathrm{T}]$, Sect. 10, where he introduces global rigid spaces, that he is to "follow fully and faithfully a plan furnished by Grothendieck". Just as schemes over a valuation ring $R$ have a generic fibre, which is a scheme over the field of fractions $K=Q(R)$, Grothendieck had the idea that formal schemes (of topologically finite type) over a complete valuation ring $R$ (of dimension 1) should admit a generic fibre over the field of fractions $K$ which, in some sense, is obtained by tensoring with $K$ over $R$. Of course, the resulting object cannot be a formal scheme any more, although it can well be interpreted as a rigid analytic space in the sense of Tate. Thus, there is a natural functor

$$
\text { Rig : (Formal } R \text {-Schemes }) \longrightarrow \text { (Rigid } K \text {-Spaces })
$$


which associates to a formal $R$-scheme of topologically finite type its generic fibre as rigid $K$-space. It was Raynaud, who studied this functor extensively; cf. [R1]. He proved the following basic result, see also [FRG1]:

Theorem. The functor Rig induces an equivalence between the following categories:

(i) the localization by "admissible" formal blowing-up of the category of quasicompact "admissible" formal R-schemes, and

(ii) the category of quasi-compact and quasi-separated rigid $K$-spaces.

At the heart of the equivalence lies the observation that the rational subdomains of affinoid spaces, which play a key role in the work of Gerritzen and Grauert mentioned above, correspond to the notion of admissible formal blowingup in the world of formal schemes, as considered by Raynaud. In fact, over an affine formal scheme $\operatorname{Spf} A$, such a blowing-up is just the formal completion of an ordinary scheme theoretic blowing up on $\operatorname{Spec} A$, with a center contained in the special fibre. Also note that this equivalence opens up a totally new path to rigid analytic spaces which, in addition, allows to generalize the concept to situations where the base field $K$ is replaced by a quite general object $S$, which, by itself, may not admit any interpretation in terms of Tate's rigid analytic spaces.

A really useful aspect of Raynaud's approach to rigid analytic spaces is the fact that it brings methods from algebraic geometry into play. To simplify things, let $K$ be a field with a complete non-Archimedean absolute value $|\cdot|$, assumed to be nontrivial. Let $R$ be its valuation ring, and fix an element $t \in R$ satisfying $0<|t|<1$. Then, given a morphism of rigid $K$-spaces $\varphi_{K}: X_{K} \longrightarrow Y_{K}$, one can look for a formal $R$-model $\varphi$ of $\varphi_{K}$, i. e., for a morphism of formal $R$-schemes $\varphi: X \longrightarrow Y$ which, under the functor Rig, is a representative of $\varphi_{K}$. Then interpreting $\varphi$ as the inductive limit of the scheme morphisms $\varphi_{i}: X_{i} \longrightarrow Y_{i}$ obtained from $\varphi$ by tensoring with $R /\left(t^{i}\right)$ over $R$, one may apply methods from algebraic geometry in order to investigate $\varphi$. At the end, information on $\varphi$ can be carried over to information on $\varphi_{K}$.

The crucial step in the just described procedure consists in constructing a suitable formal $R$-model of the morphism of rigid spaces $\varphi_{K}$, which is to be studied. 
If $\varphi_{K}: X_{K} \longrightarrow Y_{K}$ enjoys a certain property (P), applicable to morphisms of both, rigid $K$-spaces and formal $R$-schemes, one would like to construct a formal $R$-model $\varphi: X \longrightarrow Y$ enjoying $(\mathrm{P})$. As is easy to imagine, the problem will not admit a solution for any kind of property $(\mathrm{P})$. For example, looking at a smooth projective curve $C_{K} \longrightarrow$ Spec $K$ and taking for $\varphi_{K}: X_{K} \longrightarrow Y_{K}$ the morphism of rigid spaces derived from it via analytification, the theory of models for curves shows that we can at best expect a semi-stable formal $R$-model $\varphi: X \longrightarrow Y$, but not necessarily a smooth one. Thus, smoothness is too strong for these purposes. Consequently, one has to look at weaker properties (P) such as flatness. Indeed the case where $(\mathrm{P})$ means "flatness" is basic and admits a positive solution; the necessary techniques are described in [RG] and [FRG2]. Another important case is the one where $(\mathrm{P})$ means "reduced fibres". It was dealt with in [FRG3] and [FRG4], leading to a result which may be viewed as a first step towards a general semi-stable reduction theorem.

Rigid geometry in the sense of Tate and Raynaud works well for coherent sheaves. The same cannot be said for general abelian sheaves, since there are examples of such sheaves $\mathcal{F}$ on a rigid space $X_{K}$ such that all stalks $\mathcal{F}_{x}$ for $x \in X_{K}$ are trivial, without $\mathcal{F}$ being trivial itself. This is a clear indication for the fact that, in order to handle abelian sheaves and to compute their étale cohomology, rigid spaces do not contain sufficiently many points. To remedy such a fault, several concepts have been developed, which we want to mention briefly. Continuing Raynaud's point of view, it is natural to take into account all formal $R$-models $X^{\prime}$ of a given rigid space $X_{K}$ and to pass to the projective limit $\langle X\rangle=\lim X^{\prime}$, which is called the Zariski-Riemann space associated to $X_{K}$. The latter was introduced by Fujiwara in $[\mathrm{F}]$. By its definition, the Zariski-Riemann space $\langle X\rangle$ is a locally ringed space with respect to the inverse limit topology, although the latter may fail to be Hausdorff, even if $X_{K}$ is separated. There is a canonical specialization map sp: $X_{K} \longrightarrow\langle X\rangle$, which is injective and has dense image. Furthermore, one can show that sp induces an equivalence between the category of abelian sheaves on $X_{K}$ and the one on $\langle X\rangle$.

Another concept, which to a large extent is equivalent to the concept of ZariskiRiemann space, was introduced by Huber in his monography on Adic Spaces [H]. Instead of dealing with formal models and admissible formal blowing-up, Huber bases his definition on valuation spectra of certain topological rings, which can be quite general. Closer to classical rigid geometry is the approach by Berkovich 
with his non-Archimedean analytic spaces, nowadays called Berkovich spaces; see [B1], [B2]. Berkovich also works with valuation spectra, although he is restricting himself to valuations of height 1 . This means paying a little price, but the approach still works quite well for so-called overconvergent sheaves. On the other hand, topologies are quite accessible and will be Hausdorff in general. In fact, for a separated rigid space, the associated Berkovich space may be viewed as the biggest Hausdorff quotient of the corresponding Zariski-Riemann or adic space.

\section{Affinoid spaces, a first naive approach}

We will start now to discuss rigid geometry in more detail. Due to lack of space, only a few proofs can be included. For the convenience of the reader, we have chosen $[\mathrm{B}]$ as a coherent reference source, whenever possible.

Let $K$ be a field with a complete non-Archimedean absolute value $|\cdot|$ which is supposed to be non-trivial, and let $\bar{K}$ be its completed algebraic closure. We write

$$
\mathbb{B}^{n}(\bar{K})=\left\{\left(x_{1}, \ldots, x_{n}\right) \in \bar{K}^{n} ;\left|x_{i}\right| \leq 1\right\}
$$

for the closed unit ball in the $n$-dimensional affine $n$-space over $\bar{K}$ (although $\mathbb{B}^{n}(\bar{K})$ is open in $\bar{K}^{n}$ as well) and choose a set of $n$ variables $\zeta_{1}, \ldots, \zeta_{n}$ as coordinate functions on $\mathbb{B}^{n}(\bar{K})$.

Definition 2.1. Let $T_{n}=K\left\langle\zeta_{1}, \ldots, \zeta_{n}\right\rangle$ be the $K$-algebra of all power series

$$
f=\sum_{\nu \in \mathbb{N}^{n}} c_{\nu} \zeta^{\nu}=\sum_{\nu \in \mathbb{N}^{n}} c_{\nu_{1} \ldots \nu_{n}} \zeta_{1}^{\nu_{1}} \ldots \zeta_{n}^{\nu_{n}}
$$

with coefficients in $K$, which are convergent on $\mathbb{B}^{n}(\bar{K})$ or, equivalently, satisfy $\lim _{|\nu| \rightarrow \infty}\left|c_{\nu}\right|=0$. Then $T_{n}$ is called the Tate algebra of restricted power series in $n$ variables over $K$.

One may ask why algebras of power series converging on closed balls are considered, and not on open balls such as $\left\{\left(x_{1}, \ldots, x_{n}\right) \in \bar{K}^{n} ;\left|x_{i}\right|<1\right\}$. The reason is that algebras of the latter type are much more complicated to handle. Later they will be viewed as projective limits of Tate algebras, corresponding to the interpretation of open balls as an increasing union of smaller closed balls. 
A major advantage of power series on closed balls consists in the fact that their Gauß norm can be defined. On $T_{n}$ the latter is given by

$$
|f|=\max \left|c_{\nu}\right| \quad \text { for } \quad f=\sum_{\nu} c_{\nu} \zeta^{\nu}
$$

The Gauß norm is a $K$-algebra norm providing $T_{n}$ with the structure of a Banach $K$-algebra; see [B], 1.2/3. Let $R$ be the valuation ring of $K$ and $k$ its residue field. Setting

$$
T_{n}^{\circ}=\left\{f \in T_{n} ;|f| \leq 1\right\},
$$

we get the so-called reduction of $T_{n}$ by tensoring $T_{n}^{\circ}$ with $k$ over $R$, namely

$$
T_{n}^{\circ} \otimes_{R} k=k\left[\zeta_{1}, \ldots, \zeta_{n}\right],
$$

which is a polynomial ring in $n$ variables over $k$.

Via approximation arguments, Euclid's division in $k\left[\zeta_{1}, \ldots, \zeta_{n}\right]$ can be lifted to $T_{n}$, thus leading to so-called Weierstraß division here; see [B], Sect. 1.2. The latter is used as a basic tool for establishing Noether normalization for Tate algebras. From this one can conclude that $T_{n}$ is noetherian, that it is jacobson (in the sense that all nilradicals coincide with corresponding Jacobson radicals), and that for any maximal ideal $\mathfrak{m} \subset T_{n}$, the field $T_{n} / \mathfrak{m}$ is finite over $K$.

For any ideal $\mathfrak{a} \subset T_{n}$, the quotient $T_{n} / \mathfrak{a}$ is called an affinoid $K$-algebra. Just as the series $f \in T_{n}$ are viewed as analytic functions on $\mathbb{B}^{n}(\bar{K})$, we may view their residue classes in $T_{n} / \mathfrak{a}$ as analytic functions on the Zariski closed subset

$$
V(\mathfrak{a})=\left\{x \in \mathbb{B}^{n}(\bar{K}) ; g(x)=0 \text { for all } g \in \mathfrak{a}\right\} \subset \mathbb{B}^{n}(\bar{K}) .
$$

Note that, due to the fact that $T_{n}$ is jacobson, we have Hilbert's Nullstellensatz: an element $\bar{f} \in T_{n} / \mathfrak{a}$ induces the zero function on $V(\mathfrak{a})$ if and only if $\bar{f}$ is nilpotent.

Similarly as one can proceed in algebraic geometry with affine schemes (of finite type over some field $K$ ), we may view the Zariski closed subsets of type $V(\mathfrak{a}) \subset \mathbb{B}^{n}(\bar{K})$, together with their algebras of functions $T_{n} / \mathfrak{a}$, as basic local objects in rigid geometry, referred to as affinoid $K$-spaces. One can even show that the canonical map $V(\mathfrak{a}) \longrightarrow \operatorname{Max}\left(T_{n} / \mathfrak{a}\right)$ to the spectrum of maximal ideals in $T_{n} / \mathfrak{a}$ yields a bijection $V(\mathfrak{a}) / \Gamma \stackrel{\sim}{\sim} \operatorname{Max}\left(T_{n} / \mathfrak{a}\right)$ if we divide out the action of the automorphism group $\Gamma=\operatorname{Aut}(\bar{K} / K)$ on the left-hand side; see [BGR], 7.1.1/1. In particular, from this point of view a rigid $K$-space is just a pair $\operatorname{Sp} A:=(\operatorname{Max} A, A)$, where $A$ is an affinoid $K$-algebra and Max $A$ denotes its spectrum of maximal ideals. 
As far as morphisms are concerned, we may start with maps

$$
\mathbb{B}^{n}(\bar{K}) \supset V(\mathfrak{a}) \longrightarrow \mathbb{B}^{m}(\bar{K})
$$

which are given by $m$ residue classes in $\left(T_{n} / \mathfrak{a}\right)^{\circ}$, where the latter means the $R$-subalgebra of all elements $f \in T_{n} / \mathfrak{a}$, whose sup-norm

$$
|f|_{\text {sup }}=\sup \{|f(x)| ; x \in V(\mathfrak{a})\}
$$

is bounded by 1 . Note that, for Tate algebras $T_{n}$, the sup-norm coincides with the Gauß norm; see [B], 1.2/5. Furthermore, one can show that any $K$-homomorphism $\sigma: K\left\langle\zeta_{1}, \ldots, \zeta_{m}\right\rangle \longrightarrow T_{n} / \mathfrak{a}$ satisfies $\left|\sigma\left(\zeta_{i}\right)\right|_{\text {sup }} \leq 1$ for all $i$ and that, conversely, for given elements $h_{1}, \ldots, h_{m} \in T_{n} / \mathfrak{a}$ with $\left|h_{i}\right|_{\text {sup }} \leq 1$, there is a unique $K$-homomorphism $\sigma: K\left\langle\zeta_{1}, \ldots, \zeta_{m}\right\rangle \longrightarrow T_{n} / \mathfrak{a}$ satisfying $\sigma\left(\zeta_{i}\right)=h_{i}$ for all $i$; see [B], $1.4 / 18$. Therefore the morphisms $V(\mathfrak{a}) \longrightarrow \mathbb{B}^{m}(\bar{K})$ of the type just described correspond essentially to the $K$-homomorphisms $T_{m} \longrightarrow T_{n} / \mathfrak{a}$, and it is natural to define a morphism of affinoid $K$-spaces $\varphi: \operatorname{Sp} A \longrightarrow \operatorname{Sp} B$ as a pair $(\varphi, \sigma)$, where $\sigma: B \longrightarrow A$ is a $K$-homomorphism and $\varphi: \operatorname{Max} A \longrightarrow \operatorname{Max} B$ is the map $\mathfrak{m} \longmapsto \sigma^{-1}(\mathfrak{m})$. In particular, note that $\sigma^{-1}(\mathfrak{m})$ is a maximal ideal in $B$, for any maximal ideal $\mathfrak{m} \subset A$; see the explanations in [B], at the end of Sect. 1.5.

Identifying the physical points of an affinoid $K$-space with the spectrum of maximal ideals of its corresponding affinoid $K$-algebra is certainly permitted, due to Hilbert's Nullstellensatz. However, let us point out that the whole prime spectrum should not be used. Namely, the definition of reasonable open subspaces of affinoid $K$-spaces involves a combination of localization and subsequent completion on the level of affinoid $K$-algebras, as we will see below. Such a process behaves well with respect to maximal ideals, but not with respect to more general prime ideals, since for a completed localization $\tau: A \longrightarrow A^{\prime}$ there might exist prime ideals $\mathfrak{p} \subset A^{\prime}$ such that $\tau\left(\tau^{-1}(\mathfrak{p})\right) A^{\prime} \subsetneq \mathfrak{p}$.

Now, in order to introduce open subspaces of affinoid $K$-spaces, we follow Tate in applying a truely formalistic point of view:

Definition 2.2. Let $X=\operatorname{Sp} A$ be an affinoid $K$-space. A subset $U \subset X$ is called an affinoid subdomain of $X$ if there exists a morphism of affinoid $K$-spaces $\iota: X^{\prime} \longrightarrow X$ with $\iota\left(X^{\prime}\right) \subset U$ such that the following universal property holds: 
Any morphism of affinoid $K$-spaces $\varphi: Y \longrightarrow X$ satisfying $\varphi(Y) \subset U$ admits a unique factorization through $\iota: X^{\prime} \longrightarrow X$ via a morphism of affinoid $K$-spaces $\varphi^{\prime}: Y \longrightarrow X^{\prime}$.

Of course, if $U \subset X$ is an affinoid subdomain of $X$, then the corresponding morphism $\iota: X^{\prime} \longrightarrow X$, as required in the definition, is uniquely determined by $U$. Furthermore, it is not too hard to show that, pointwise, $\iota$ induces a bijection from $X^{\prime}$ onto $U$; see [B], 1.6/10. Thus, any affinoid subdomain $U \subset X$ is automatically equipped with a unique structure of affinoid $K$-space. In order to exhibit explicit examples of affinoid subdomains, let us specify some classes of subsets of affinoid $K$-spaces.

Definition 2.3. Let $X=\operatorname{Sp} A$ be an affinoid $K$-space.

(i) A subset in $X$ of type

$$
X\left(f_{1}, \ldots, f_{r}\right)=\left\{x \in X ;\left|f_{i}(x)\right| \leq 1\right\}
$$

for functions $f_{1}, \ldots, f_{r} \in A$ is called a Weierstraß domain in $X$.

(ii) A subset in $X$ of type

$$
X\left(f_{1}, \ldots, f_{r}, g_{1}^{-1}, \ldots, g_{s}^{-1}\right)=\left\{x \in X ;\left|f_{i}(x)\right| \leq 1,\left|g_{j}(x)\right| \geq 1\right\}
$$

for functions $f_{1}, \ldots, f_{r}, g_{1}, \ldots, g_{s} \in A$ is called a Laurent domain in $X$.

(iii) A subset in $X$ of type

$$
X\left(\frac{f_{1}}{f_{0}}, \ldots, \frac{f_{r}}{f_{0}}\right)=\left\{x \in X ;\left|f_{i}(x)\right| \leq\left|f_{0}(x)\right|\right\}
$$

for functions $f_{0}, \ldots, f_{r} \in A$ without common zeros is called a rational domain in $X$.

Proposition 2.4. Weierstraß, Laurent, and rational domains are examples of affinoid subdomains.

For a proof, see $[\mathrm{B}], 1.6 / 11$. Let us just mention that for a Weierstraß domain $X\left(f_{1}, \ldots, f_{r}\right) \subset X$ the corresponding affinoid $K$-algebra is given by

$$
A\left\langle f_{1}, \ldots, f_{r}\right\rangle=A\left\langle\zeta_{1}, \ldots, \zeta_{r}\right\rangle /\left(\zeta_{i}-f_{i} ; i=1, \ldots, r\right),
$$


for a Laurent domain $X\left(f_{1}, \ldots, f_{r}, g_{1}^{-1}, \ldots, g_{s}^{-1}\right) \subset X$ by

$$
\begin{aligned}
A\left\langle f, g^{-1}\right\rangle & =A\left\langle f_{1}, \ldots, f_{r}, g_{1}^{-1}, \ldots, g_{s}^{-1}\right\rangle \\
& =A\left\langle\zeta_{1}, \ldots, \zeta_{r}, \xi_{1}, \ldots, \xi_{s}\right\rangle /\left(\zeta_{i}-f_{i}, 1-g_{j} \xi_{j} ; i=1, \ldots, r ; j=1, \ldots, s\right),
\end{aligned}
$$

and for a rational domain $X\left(\frac{f_{1}}{f_{0}}, \ldots, \frac{f_{r}}{f_{0}}\right) \subset X$ by

$$
A\left\langle\frac{f_{1}}{f_{0}}, \ldots, \frac{f_{r}}{f_{0}}\right\rangle=A\left\langle\zeta_{1}, \ldots, \zeta_{r}\right\rangle /\left(f_{i}-f_{0} \zeta_{i} ; i=1, \ldots, r\right)
$$

It is clear that any Weierstraß domain in $X$ is also Laurent. Furthermore, one can show that Laurent domains in $X$ are rational. Namely they can be viewed as finite intersections of rational domains, and any such intersection is rational again; see [B], 1.6/14. Rational domains are not yet mentioned in Tate's notes [T]. However, they appear quite naturally. If we consider a Laurent domain $X^{\prime} \subset X$ and a Weierstraß domain $X^{\prime \prime} \subset X^{\prime}$, then, in general, $X^{\prime \prime}$ will be neither Weierstraß nor Laurent in $X$. However, we can see that $X^{\prime \prime}$ is rational in $X$. Furthermore, one knows that any rational domain in $X$ occurs in this way; see [B], 1.6/16 and $1.6 / 17$.

It should be noted that any affinoid $K$-space $X=\operatorname{Sp} A$ carries a natural topology, which is induced from the absolute value of $K$ or its (unique) extension to $\bar{K}$. Indeed, $\mathbb{B}^{n}(\bar{K})$ is a topological space this way, and so are its (Zariski) closed subspaces $V(\mathfrak{a})$, for any ideal $\mathfrak{a} \subset T_{n}$. Identifying the spectrum of maximal ideals $\operatorname{Max} A$, where $A=T_{n} / \mathfrak{a}$, with the quotient $V(\mathfrak{a}) / \Gamma$ for $\Gamma=\operatorname{Aut}(\bar{K} / K)$, we may consider on the point set of $\operatorname{Sp} A$ the quotient topology of the one on $V(\mathfrak{a})$. The resulting topology is called the canonical topology on $\mathrm{Sp} A$, and one knows ([B], 1.6/2 and 1.6/19):

Proposition 2.5. Any affinoid subdomain of an affinoid $K$-space $X$ is open with respect to the canonical topology on $X$. The Weierstraß domains form a basis of this topology.

More precise information on the structure of general affinoid subdomains is provided by the following result ([B], 1.8/12): 
Theorem 2.6 (Gerritzen - Grauert [GG]). Let $X$ be an affinoid $K$-space and $U \subset X$ an affinoid subdomain. Then $U$ is a finite union of rational subdomains of $X$.

The proof of the Theorem in $[\mathrm{GG}]$ provides quite precise information on the functions needed to describe the affinoid subdomains covering a given affinoid subdomain $U \subset X$. Surprisingly, a more rapid, but less specific proof was recently given by Temkin [Te1] from the viewpoint of Berkovich theory.

Using the fact that affinoid subdomains carry a well-defined structure of affinoid $K$-space, we are able now to introduce the sheaf of locally analytic functions on any affinoid $K$-space $X=\operatorname{Sp} A$. Indeed, associating to an affinoid subdomain $U \subset X$ its corresponding affinoid $K$-algebra constitutes a presheaf on a basis of the canonical topology on $X$. The associated sheaf is called the sheaf of locally analytic functions on $X$. However, since $X$ is totally disconnected with respect to the canonical topology, the algebra of global sections on $X$ will be substantially larger than the affinoid $K$-algebra $A$ giving rise to the definition of $X$, except for trivial situations.

\section{Tate's Acyclicity Theorem}

Although it is possible to define the sheaf of locally analytic functions on an affinoid $K$-space in a natural way, as we have just seen, we are facing the problem that this sheaf has too many sections. For example, we expect a function, which is analytic in any reasonable sense on a unit ball $\mathbb{B}^{n}=\operatorname{Sp} T_{n}$, to admit a globally convergent power series expansion and, thus, to correspond to an element in $T_{n}$. However, the class of locally analytic functions does not behave this way. For example, characteristic functions of open subballs $U \subset \mathbb{B}^{n}$ are locally analytic on $\mathbb{B}^{n}$, but cannot be represented by series in $T_{n}$, unless $U=\mathbb{B}^{n}$.

But how to set up a reasonable notion of local analyticity, without abandoning the concept that, on nice spaces such as balls, locally analytic functions admit globally convergent power series expansions? The fundamental idea pursued by Tate is to restrict the class of open coverings which are allowed for testing local analyticity. To be more precise, consider an affinoid $K$-space $X=\operatorname{Sp} A$ and denote by $\mathcal{O}_{X}$ the presheaf of affinoid functions on $X$. Thereby we mean the 
functor on the category of affinoid subdomains $U \subset X$ which associates to any such $U$ its corresponding affinoid $K$-algebra $A_{U}$. Then we may ask if there exists a reasonable class of coverings $\mathfrak{U}=\left(U_{i}\right)_{i \in I}$ of $X$ by affinoid subdomains $U_{i} \subset X$ such that the associated diagram

$$
\begin{aligned}
\mathcal{O}_{X}(U) \rightarrow \prod_{i \in I} \mathcal{O}_{X}\left(U_{i}\right) & \rightrightarrows \prod_{i, j \in I} \mathcal{O}_{X}\left(U_{i} \cap U_{j}\right), \\
f \longmapsto\left(\left.f\right|_{U_{i}}\right)_{i \in I}, \quad\left(f_{i}\right)_{i \in I} & \longmapsto\left\{\begin{array}{c}
\left(\left.f_{i}\right|_{U_{i} \cap U_{j}}\right)_{i, j \in I} \\
\left(\left.f_{j}\right|_{U_{i} \cap U_{j}}\right)_{i, j \in I}
\end{array}\right.
\end{aligned}
$$

is always exact (note that the intersection of two affinoid subdomains in $X$ is an affinoid subdomain in $X$ again by [B], 1.6/14). The answer is given by the following result, which is part of Tate's Acyclicity Theorem.

Theorem 3.1 (Tate). Let $X$ be an affinoid $K$-space and $\mathfrak{U}=\left(U_{i}\right)_{i \in I}$ a finite covering of $X$ by affinoid subdomains $U_{i} \subset X$. Then the above diagram (*) is exact.

We can proceed one step further and consider the augmented Čech complex

$$
0 \longrightarrow \mathcal{F}(X) \stackrel{\varepsilon}{\longrightarrow} C^{0}(\mathfrak{U}, \mathcal{F}) \stackrel{d^{0}}{\longrightarrow} C^{1}(\mathfrak{U}, \mathcal{F}) \stackrel{d^{1}}{\longrightarrow} \ldots
$$

for any covering $\mathfrak{U}=\left(U_{i}\right)_{i \in I}$ of $X$ by affinoid subdomains $U_{i} \subset X$ and any presheaf $\mathcal{F}$ on the category of affinoid subdomains of $X$. If the sequence is exact, $\mathfrak{U}$ is called $\mathcal{F}$-acyclic. Using this terminology, the full version of Tate's Acyclicity Theorem reads as follows:

Theorem 3.2 (Tate). Let $X$ be an affinoid $K$-space and $\mathfrak{U}$ a finite covering of $X$ by affinoid subdomains. Then $\mathfrak{U}$ is acyclic for the presheaf $\mathcal{O}_{X}$ of affinoid functions on $X$.

For a complete proof we refer to [B], Sect. 1.9. The strategy consists in simplifying the affinoid covering $\mathfrak{U}$ as much as possible, with the help of some general facts about Čech cohomology. Moreover, it is enough to consider the Čech complex of alternating cochains. Then, for $X=\operatorname{Sp} A$, it remains to actually do the proof in the case where $\mathfrak{U}$ is a Laurent covering generated by a single function 
$f \in A$; i. e., where

$$
\mathfrak{U}=\left(X(f), X\left(f^{-1}\right)\right) .
$$

Thus, we have to show that the sequence

$$
\begin{gathered}
0 \longrightarrow A \stackrel{\varepsilon}{\longrightarrow} A\langle f\rangle \times A\left\langle f^{-1}\right\rangle \stackrel{\delta}{\longrightarrow} A\left\langle f, f^{-1}\right\rangle \longrightarrow 0 \\
f \stackrel{\varepsilon}{\longmapsto}\left(\left.f\right|_{X(f)},\left.f\right|_{X\left(f^{-1}\right)}\right),\left.\quad(f, g) \stackrel{\delta}{\longmapsto} f\right|_{X\left(f, f^{-1}\right)}-\left.g\right|_{X\left(f, f^{-1}\right)},
\end{gathered}
$$

is exact. The sequence is part of the following commutative diagram:

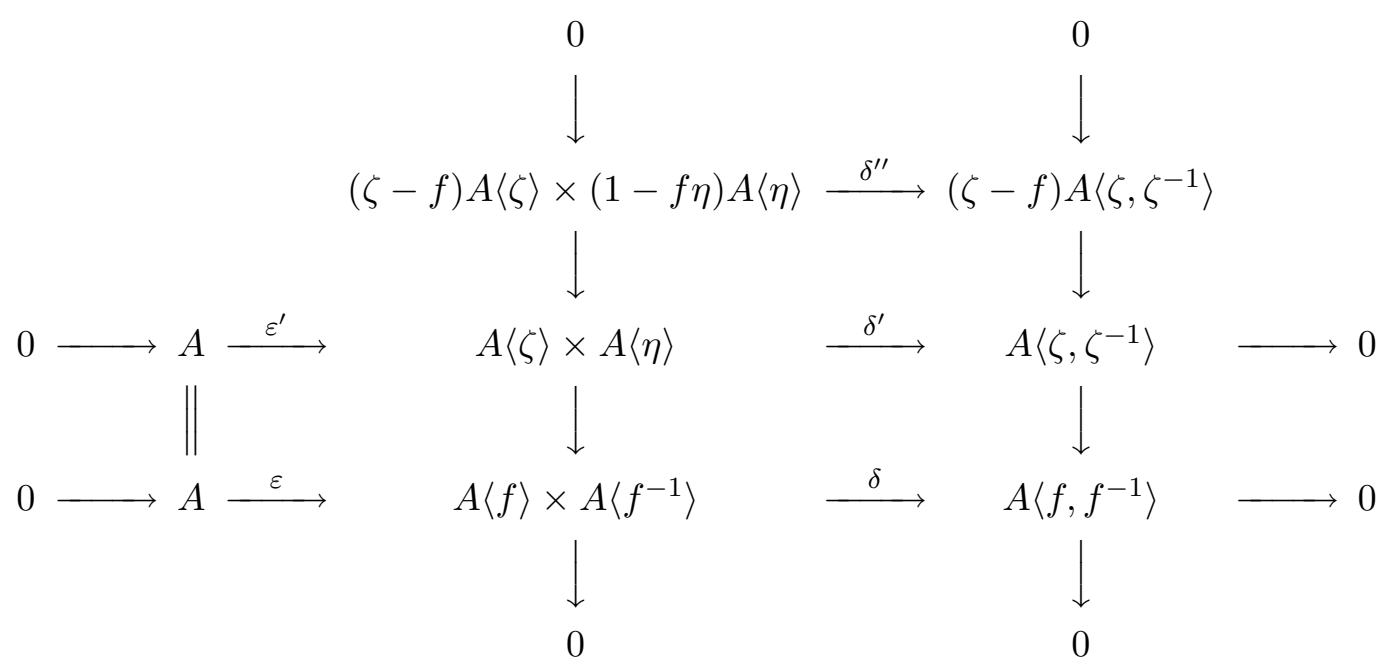

The symbols $\zeta, \eta$ denote indeterminates, $\varepsilon^{\prime}$ is the canonical injection, $\delta^{\prime}$ is given by $\left(h_{1}(\zeta), h_{2}(\eta)\right) \longmapsto h_{1}(\zeta)-h_{2}\left(\zeta^{-1}\right)$, and $\delta^{\prime \prime}$ is induced by $\delta^{\prime}$. Furthermore, the vertical maps are characterized by $\zeta \longmapsto f$ and $\eta \longmapsto f^{-1}$, respectively. The first column of the diagram is exact due to the definition of $A\langle f\rangle$ and $A\left\langle f^{-1}\right\rangle$. Also the second column is exact since

$$
\begin{aligned}
A\left\langle f, f^{-1}\right\rangle & =A\langle\zeta, \eta\rangle /(\zeta-f, 1-f \eta) \\
& =A\langle\zeta, \eta\rangle /(\zeta-f, 1-\zeta \eta)=A\left\langle\zeta, \zeta^{-1}\right\rangle /(\zeta-f) .
\end{aligned}
$$

Clearly, $\delta^{\prime}$ is surjective. Since

$$
\begin{aligned}
(\zeta-f) A\left\langle\zeta, \zeta^{-1}\right\rangle & =(\zeta-f) A\langle\zeta\rangle \oplus(\zeta-f) \zeta^{-1} A\left\langle\zeta^{-1}\right\rangle \\
& =(\zeta-f) A\langle\zeta\rangle \oplus\left(1-f \zeta^{-1}\right) A\left\langle\zeta^{-1}\right\rangle,
\end{aligned}
$$

we see that $\delta^{\prime \prime}$ is bijective. Furthermore, the second row is exact, since

$$
0=\delta^{\prime}\left(\sum_{i=0}^{\infty} a_{i} \zeta^{i}, \sum_{i=0}^{\infty} b_{i} \eta^{i}\right)=\sum_{i=0}^{\infty} a_{i} \zeta^{i}-\sum_{i=0}^{\infty} b_{i} \eta^{i}
$$


implies $a_{i}=b_{i}=0$ for $i>0$ and $a_{0}-b_{0}=0$. Finally, looking at the third row, the exactness follows with the aide of the snake lemma, applied to the diagram of the two vertical exact sequences.

For an affinoid $K$-space $X=\operatorname{Sp} A$ and an $A$-module $M$, we can consider the presheaf $M \otimes_{A} \mathcal{O}_{X}$ on the category of affinoid subdomains of $X$ which is given by

$$
U \longmapsto M \otimes_{A} \mathcal{O}_{X}(U) .
$$

A simple argument, see [B], 1.9/10, shows that the assertion of Tate's Acyclicity Theorem can be generalized to such a presheaf in place of $\mathcal{O}_{X}$ :

Corollary 3.3. Let $X=\operatorname{Sp} A$ be an affinoid $K$-space, $M$ an A-module, and $\mathfrak{U}$ a finite covering of $X$ by affinoid subdomains. Then $\mathfrak{U}$ is acyclic for the presheaf $M \otimes_{A} \mathcal{O}_{X}$

\section{Rigid analytic spaces}

Let $X$ be an affinoid $K$-space and $U \subset X$ a subset which is open with respect to the canonical topology. We might call $U$ admissible open in $X$ if it is an affinoid subdomain of $X$ (note that affinoid subdomains are always open in $X$, due to 2.5). Furthermore, a covering $U=\bigcup_{i \in I} U_{i}$ of an admissible open subset $U \subset X$ might be called admissible if it is a finite covering by admissible open subsets $U_{i} \subset X$. Thus, the presheaf $\mathcal{O}_{X}$ of affinoid functions on $X$ is defined on the category of all admissible open subsets of $X$, and Tate's Acyclicity Theorem in the version of 3.1 states that $\mathcal{O}_{X}$ is, in fact, a sheaf on $X$ with respect to admissible open coverings. In a certain sense, we will view $\mathcal{O}_{X}$ as the structure sheaf of the affinoid $K$-space $X$. To be more precise, we need the notion of a Grothendieck topology $[\mathrm{A}]$, adapted to our situation. It generalizes the notion of a topology.

Definition 4.1. A Grothendieck topology $\mathfrak{T}$ on a set $X$ consists of a category Cat $\mathfrak{T}$ of subsets in $X$, with inclusions as morphisms, and a set Cov $\mathfrak{T}$ of families $\left(U_{i} \longrightarrow U\right)_{i \in I}$ of morphisms in Cat $\mathfrak{T}$ satisfying $U=\bigcup_{i \in I} U_{i}$, called coverings, such that the following hold:

(1) If $\Phi: U \longrightarrow V$ is an isomorphism in Cat $\mathfrak{T}$, then $(\Phi) \in \operatorname{Cov} \mathfrak{T}$. 
(2) If $\left(U_{i} \longrightarrow U\right)_{i \in I}$ and $\left(V_{i j} \longrightarrow U_{i}\right)_{j \in J_{i}}$ for $i \in I$ belong to Cov $\mathfrak{T}$, then the same is true for the composition $\left(V_{i j} \longrightarrow U_{i} \longrightarrow U\right)_{i \in I, j \in J_{i}}$.

(3) If $\left(U_{i} \longrightarrow U\right)_{i \in I}$ is in $\operatorname{Cov} \mathfrak{T}$ and if $V \longrightarrow U$ is a morphism in Cat $\mathfrak{T}$, then the fibred products $U_{i} \times_{U} V=U_{i} \cap V$ exist in Cat $\mathfrak{T}$, and $\left(U_{i} \times_{U} V \longrightarrow V\right)_{i \in I}$ belongs to $\operatorname{Cov} \mathfrak{T}$.

The objects of Cat $\mathfrak{T}$ are referred to as the admissible open sets of $X$, assuming tacidly that we provide $X$ with the topology generated by all these sets. Likewise, the elements of Cov $\mathfrak{T}$ are the admissible open coverings of $X$. As indicated above, we are particularly interested in the so-called weak Grothendieck topology $\mathfrak{T}_{X}$ on affinoid $K$-spaces $X$, where Cat $\mathfrak{T}_{X}$ is the category of affinoid subdomains in $X$, and $\operatorname{Cov} \mathfrak{T}_{X}$ the set of all finite coverings of affinoid subdomains in $X$ by sets of the same type. One knows that any morphism of affinoid $K$-spaces $\varphi: Z \longrightarrow X$ is continuous with respect to the weak Grothendieck topology on $X$ and $Z$, in the sense that $\varphi$-inverses of admissible open sets and coverings of $\mathfrak{T}_{X}$ are admissble open with respect to $\mathfrak{T}_{Z}$. This follows from the fact that $\varphi^{-1}(U)$ is an affinoid subdomain in $Z$, for any affinoid subdomain $U \subset X$; see [B], 1.6/13.

A presheaf with respect to a Grothendieck topology $\mathfrak{T}$ is a contravariant functor $\mathcal{F}$ on Cat $\mathfrak{T}$ with values in some category $\mathfrak{C}$. Such a functor is called a sheaf if the diagram

$$
\mathcal{F}(U) \rightarrow \prod_{i \in I} \mathcal{F}\left(U_{i}\right) \rightrightarrows \prod_{i, j \in I} \mathcal{F}\left(U_{i} \times_{U} U_{j}\right)
$$

is exact for every covering $\left(U_{i} \longrightarrow U\right)_{i \in I}$ in $\operatorname{Cov} \mathfrak{T}$ (assuming that $\mathfrak{C}$ admits cartesian products). Thus 3.1 just says that, for an affinoid $K$-space $X$, the functor $\mathcal{O}_{X}$, which associates to an affinoid subdomain $U \subset X$ its corresponding affinoid $K$-algebra, is a sheaf with respect to the weak Grothendieck topology on $X$.

There is a canonical way to enlarge the weak Grothendieck topology on affinoid $K$-spaces by adding more admissible open sets and more admissible coverings in such a way that morphisms of affinoid $K$-spaces remain continuous and sheaves extend uniquely to sheaves with respect to this new topology. The resulting Grothendieck topology is the strong Grothendieck topology on affionid $K$-spaces which we define now. 
Definition 4.2. Let $X$ be an affinoid $K$-space. The strong Grothendieck topology on $X$ is given as follows.

(i) A subset $U \subset X$ is called admissible open if there is a (not necessarily finite) covering $U=\bigcup_{i \in I} U_{i}$ of $U$ by affinoid subdomains $U_{i} \subset X$ such that for all morphisms of affinoid $K$-spaces $\varphi: Z \longrightarrow X$ satisfying $\varphi(Z) \subset U$ the covering $\left(\varphi^{-1}\left(U_{i}\right)\right)_{i \in I}$ of $Z$ admits a refinement, which is a finite covering of $Z$ by affinoid subdomains.

(ii) A covering $V=\bigcup_{j \in J} V_{j}$ of some admissible open subset $V \subset X$ by means of admissible open sets $V_{j}$ is called admissible if for each morphism of affinoid $K$-spaces $\varphi: Z \longrightarrow X$ satisfying $\varphi(Z) \subset V$ the covering $\left(\varphi^{-1}\left(V_{j}\right)\right)_{j \in J}$ of $Z$ admits a refinement, which is a finite covering of $Z$ by affinoid subdomains.

Note that any covering $\left(U_{i}\right)_{i \in I}$ as in (i) is admissible by (ii). It is easily checked that the strong Grothendieck topology on $X$ really is a Grothendieck topology, and that any finite union of affinoid subdomains of $X$ is admissible open. Furthermore, one knows that Zariski open subsets of $X$ are admissible open, and that each Zariski open covering of such a subset is admissible; see [B], 1.10/9. Thus, we can say that the strong Grothendieck topology on $X$ is finer than the Zariski topology.

If $\mathcal{F}$ is a presheaf with respect to the strong Grothendieck topology on an affinoid $K$-space $X$, we write as usual

$$
\mathcal{F}_{x}=\varliminf_{\longrightarrow} \mathcal{F}(U),
$$

for the stalk of $\mathcal{F}$ at a point $x \in X$, where the limit extends over all admissible open subsets $U \subset X$ containing $x$. One knows that the stalks $\mathcal{O}_{X, x}$ of the sheaf of affinoid functions on $X$ are local $K$-algebras ([B], 1.7/1).

For any set $X$ with a Grothendieck topology $\mathfrak{T}$ on it and a sheaf $\mathcal{O}_{X}$ of $K$-algebras with respect to $\mathfrak{T}$, we call the pair $\left(X, \mathcal{O}_{X}\right)$ a ringed $K$-space. Furthermore, we talk about a locally ringed $K$-space if all stalks of $\mathcal{O}_{X}$ are local. In particular, for any affinoid $K$-space, we can consider its associated locally ringed $K$-space $\left(X, \mathcal{O}_{X}\right)$, assuming tacidly that affinoid $K$-spaces are always equipped with their strong Grothendieck topology. Using the appropriate notion of morphisms between such spaces (see [B], 1.12/1), it is clear that any morphism of affinoid $K$-spaces $\varphi: X \longrightarrow Y$ induces in a natural way a morphism of locally 
ringed $K$-spaces $\left(\varphi, \varphi^{*}\right):\left(X, \mathcal{O}_{X}\right) \longrightarrow\left(Y, \mathcal{O}_{Y}\right)$. One can show by the usual argument $([\mathrm{B}], 1.12 / 2)$ :

Proposition 4.3. The functor $X \longmapsto\left(X, \mathcal{O}_{X}\right)$ from the category of affinoid $K$-spaces to the category of locally ringed $K$-spaces is fully faithful. Thus, the former category can be viewed as a full subcategory of the latter.

Now it is more or less straightforward how to define the category of global rigid $K$-spaces. We could just say that such a rigid analytic space is a locally ringed $K$-space $\left(X, \mathcal{O}_{X}\right)$ with a Grothendieck topology on $X$ such that $X$ admits an admissible open covering $\left(X_{i}\right)_{i \in I}$ where $\left(X_{i},\left.\mathcal{O}_{X}\right|_{X_{i}}\right)$ is affinoid for all $i \in I$. However, if we proceed like this, a similar effect will occur, as we have encountered when passing from the weak to the strong Grothendieck topology on affinoid $K$-spaces: in general, it will be possible to introduce additional open sets and open coverings of global type, without changing sheaves on $X$ and without changing the structure of the defining affinoid pieces $X_{i}$. To remedy this, we observe that the strong Grothendieck topology on affinoid $K$-spaces satisfies certain completeness conditions, namely:

Proposition 4.4. Let $X$ be an affinoid $K$-space. Then:

$\left(\mathrm{G}_{0}\right) \emptyset$ and $X$ are admissible open.

$\left(\mathrm{G}_{1}\right)$ Let $\left(U_{i}\right)_{i \in I}$ be an admissible covering of an admissible open subset $U \subset X$. Furthermore, let $V \subset U$ be a subset such that $V \cap U_{i}$ is admissible open for all $i \in I$. Then $V$ is admissible open in $X$.

$\left(\mathrm{G}_{2}\right)$ Let $\left(U_{i}\right)_{i \in I}$ be a covering of an admissible open set $U \subset X$ by admissible open subsets $U_{i} \subset X$ which admits an admissible covering of $U$ as refinement. Then $\left(U_{i}\right)_{i \in I}$ itself is admissible.

If $X$ is a set with a Grothendieck topology $\mathfrak{T}$ on it satisfying the above conditions $\left(\mathrm{G}_{0}\right),\left(\mathrm{G}_{1}\right)$, and $\left(\mathrm{G}_{2}\right)$, and if $\left(X_{i}\right)_{i \in I}$ is an admissible open covering of $X$, then the Grothendieck topology on $X$ can be recovered from the ones induced on the spaces $X_{i}([\mathrm{~B}], 1.10 / 10)$. Even better, if $X$ is a set admitting a covering 
$X=\bigcup_{i \in I} X_{i}$ and if each $X_{i}$ is equipped with a Grothendieck topology satisfying $\left(\mathrm{G}_{0}\right),\left(\mathrm{G}_{1}\right)$, and $\left(\mathrm{G}_{2}\right)$, compatible in the sense that all intersections $X_{i} \cap X_{j}$ are admissible open in $X_{i}$ and $X_{j}$ and that their topologies restrict to the same Grothendieck topology on $X_{i} \cap X_{j}$, then there is a unique Grothendieck topology on $X$ satisfying $\left(\mathrm{G}_{0}\right),\left(\mathrm{G}_{1}\right)$, and $\left(\mathrm{G}_{2}\right)$, and containing all $X_{i}$ as admissible open subspaces $([\mathrm{B}], 1.10 / 11)$. Therefore it makes sense to put the definition of global rigid spaces as follows:

Definition 4.5. A rigid analytic $K$-space is a locally ringed $K$-space $\left(X, \mathcal{O}_{X}\right)$ with respect to a Grothendieck topology on $X$ such that

(i) the Grothendieck topology of $X$ satisfies conditions $\left(\mathrm{G}_{0}\right),\left(\mathrm{G}_{1}\right)$, and $\left(\mathrm{G}_{2}\right)$ of Proposition 4.4, and

(ii) $X$ admits an admissible open covering $\left(X_{i}\right)_{i \in I}$ where $\left(X_{i},\left.\mathcal{O}_{X}\right|_{X_{i}}\right)$ is an affinoid $K$-space for all $i \in I$.

$A$ morphism of rigid $K$-spaces $\left(X, \mathcal{O}_{X}\right) \longrightarrow\left(Y, \mathcal{O}_{Y}\right)$ is a morphism in the sense of locally ringed $K$-spaces.

Due to the fact that we require the completeness conditions $\left(G_{0}\right),\left(G_{1}\right)$, and $\left(\mathrm{G}_{2}\right)$, we can conclude from the properties mentioned above that global rigid $K$-spaces can be constructed in the usual way by glueing local affinoid parts. In particular, we thereby see that the category of rigid $K$-spaces admits fiber products. Namely, the completed tensor product of affinoid $K$-algebras, as dealt with in [BGR], 3.1.1 and 6.1.1/10, provides a fiber product

$$
\operatorname{Sp} A \times_{\mathrm{Sp} B} \operatorname{Sp} C=\operatorname{Sp}\left(A \hat{\otimes}_{B} C\right)
$$

in the category of affinoid $K$-spaces, and one can construct fibre products of more general type via the usual glueing process, see [BGR], 9.3.5/2.

A similar application of the glueing techniques for rigid spaces shows that we can define the analogue of Serre's GAGA-functor [S], which associates to any $K$-scheme $Z$ of locally finite type a rigid $K$-space $Z^{\text {rig }}$, called the rigid analytification of $Z$; see $[\mathrm{B}], 1.13$. Indeed, for the affine $n$-space $\mathbb{A}_{K}^{n}$ one defines its rigid analytification by glueing an increasing sequence of $n$-dimensional balls. In principle, the same procedure applies to Zariski closed subschemes of $\mathbb{A}_{K}^{n}$, thus leading to the rigid analytification of affine $K$-schemes of finite type. This makes 
it possible to deal with the general case of a $K$-scheme of locally finite type $Z$ by glueing the analytifications of open affine parts of $Z$.

Of course, there is the question of whether or not the results of Serre's paper [S] remain true in the rigid analytic setting. Generally speaking, the answer is yes, due to work of Kiehl [K1], [K2], and Köpf [Kö]. It was Kiehl who developed the theory of coherent modules on rigid $K$-spaces (see also [B], 1.14) and proved the analogues of Theorems A and B [K1], well-known from complex analysis. Furthermore, he introduced the notion of proper morphisms in rigid geometry and established the Proper Mapping Theorem; [K2], see also [B], 1.16 and 1.17. Based on this work, Köpf [Kö] was able to carry over the results from [S], in particular the algebraization of coherent analytic scheaves on the analytification of a proper $K$-scheme.

There is further work concentrating on carrying over several fundamental results from complex analysis to the context of rigid geometry. As an example, let us just mention the theme of analytic continuation such as the work of Bartenwerfer [Ba] involving Hartogs figures and of Lütkebohmert [L1].

Besides that, special questions from algebraic geometry have influenced the development of rigid geometry substantially. Tate's elliptic curves have been generalized by Mumford to curves of higher genus [M1] and to abelian varieties of higher dimension [M2], ideas which play an important role in the book of Faltings and Chai $[\mathrm{FC}]$ for the compactification of moduli spaces of abelian varieties. Raynaud [R2] has worked on the uniformization of abelian varieties and their duals, later completed by Lütkebohmert and the author [BL3]. There are even proofs in terms of rigid geometry of the semi-stable reduction theorems for curves and abelian varieties [BL1], [BL2]. Finally, let us also mention that Bertapelle [Bp] has established the technique of Weil restriction in rigid geometry.

\section{Rigid spaces as generic fibres of formal schemes}

To approach rigid geometry via formal schemes, the base field $K$, which has been used so far, is replaced by its valuation ring, which will be denoted by $R$. Thus, one starts out from the $R$-algebra $R\left\langle\zeta_{1}, \ldots, \zeta_{n}\right\rangle$ of restricted power series in a set of variables $\zeta_{i}$ with coefficients in $R$, viewing it as an $R$-model of the full algebra $T_{n}=K\left\langle\zeta_{1}, \ldots, \zeta_{n}\right\rangle$ of restricted power series with coefficients in $K$, in the sense 
that

$$
K\left\langle\zeta_{1}, \ldots, \zeta_{n}\right\rangle=R\left\langle\zeta_{1}, \ldots, \zeta_{n}\right\rangle \otimes_{R} K
$$

To justify this equation, interpret the tensor product with $K$ over $R$ as localization by $S=R-\{0\}$. Then there are inclusions

$$
R\left\langle\zeta_{1}, \ldots, \zeta_{n}\right\rangle \subset S^{-1}\left(R\left\langle\zeta_{1}, \ldots, \zeta_{n}\right\rangle\right) \subset K\left\langle\zeta_{1}, \ldots, \zeta_{n}\right\rangle
$$

and, since any series in $K\left\langle\zeta_{1}, \ldots, \zeta_{n}\right\rangle$ has bounded coefficients, the inclusion on the right is an equality.

Next consider an affinoid $K$-algebra $A_{K}=T_{n} / \mathfrak{a}$, for some ideal $\mathfrak{a} \subset T_{n}$. Then $A=R\left\langle\zeta_{1}, \ldots, \zeta_{n}\right\rangle / \mathfrak{a}^{\prime}$ with $\mathfrak{a}^{\prime}=\mathfrak{a} \cap R\left\langle\zeta_{1}, \ldots, \zeta_{n}\right\rangle$ certainly is an $R$-model of $A_{K}$. However, although $T_{n}$ is noetherian and, thus, $\mathfrak{a}$ is finitely generated, we do not know right away if the same is true for the ideal $\mathfrak{a}^{\prime} \subset R\left\langle\zeta_{1}, \ldots, \zeta_{n}\right\rangle$. Namely, $R\left\langle\zeta_{1}, \ldots, \zeta_{n}\right\rangle$ is noetherian only if the absolute value of $K$ is discrete and, thus, $R$ is a discrete valuation ring. Let us fix an ideal $I \subset R$, generated by some element $t \in R$, where $0<|t|<1$, so that the topology of $R$ coincides with the $I$-adic one.

Definition 5.1. A topological R-algebra $A$ is called

(i) of topologically finite type if it is isomorphic to an R-algebra of type $R\left\langle\zeta_{1}, \ldots, \zeta_{n}\right\rangle / \mathfrak{a}$, endowed with the I-adic topology.

(ii) of topologically finite presentation if, in addition to (i), a is finitely generated, and

(iii) admissible if, in addition to (i) and (ii), A does not have I-torsion.

From work of Raynaud and Gruson [RG], one can derive the following fundamental fact (see also [B], 2.3/4 and 2.3/5 for an elementary proof):

Proposition 5.2. Let $A$ be an R-algebra of topologically finite type. If $A$ has no I-torsion, $A$ is of topologically finite presentation and, thus, admissible.

In particular, this implies that the ideal $\mathfrak{a}^{\prime}=\mathfrak{a} \cap R\left\langle\zeta_{1}, \ldots, \zeta_{n}\right\rangle$ considered above is, indeed, finitely generated. In particular, looking for $R$-models $A$ (of topologically finite type) of an affinoid $K$-algebra $A_{K}$, we may always assume that $A$ does not admit $I$-torsion. Then $A$ is automatically of topologically finite 
presentation and, hence, admissible. Thus, the category of admissible $R$-algebras is a good one to look for $R$-models of affinoid $K$-algebras.

Next, let us consider an $R$-algebra as above, or more generally, an $R$-algebra $A$ which is complete and separated with respect to the $I$-adic topology. The latter is equivalent to the fact that the canonical morphism $A \longrightarrow \lim _{n} A /\left(I^{n}\right)$ is an isomorphism. The formal $R$-scheme $\operatorname{Spf} A$ associated to $A$ is the locally ringed space $\left(X, \mathcal{O}_{X}\right)$, where $X$ is a (true) topological space, namely the prime spectrum Spec $A /(I)$ endowed with the Zariski topology, and where $\mathcal{O}_{X}$ is the sheaf on $X$ extending the functor of topological $R$-algebras

$$
D(f) \longmapsto A\left\langle f^{-1}\right\rangle:={\underset{\varliminf}{n}}_{\lim _{n}} A /\left(I^{n}\right)\left[f^{-1}\right], \quad f \in A,
$$

given on the basic open subsets $D(f) \subset X$; see [B], 2.2. As usual, $D(f)$ denotes the Zariski open locus in $X$ where $f$ does not vanish. Note that there is a canonical isomorphism $A\langle\zeta\rangle /(1-f \zeta) \stackrel{\sim}{\sim} A\left\langle f^{-1}\right\rangle,[\mathrm{B}], 2.1 / 9$.

In the following, let us use the term formal $R$-scheme for a locally ringed space $\left(X, \mathcal{O}_{X}\right)$ which locally looks like a formal $R$-scheme of type $\operatorname{Spf} A$, as just discussed.

Definition 5.3. Let $X$ be a formal $R$-scheme. $X$ is called locally of topologically finite type (resp. locally of topologically finite presentation, resp. admissible) if there is an open affine covering $\left(U_{j}\right)_{j \in J}$ of $X$ with $U_{j}=\operatorname{Spf} A_{j}$ where $A_{j}$ is an R-algebra of topologically finite type (resp. of topologically finite presentation, resp. an admissible $R$-algebra).

Let us mention that the property of a formal $R$-scheme to be locally of topologically finite type or presentation, or to be admissible, is independent of the covering occurring in the definition; see [B], 2.4/2. In particular, an affine formal $R$-scheme $\operatorname{Spf} A$ is of this type if and only if $A$ is of topologically finite type or presentation, or is admissible.

To simplify our terminology, we will assume in the following that all formal $R$-schemes are at least locally of topologically finite type, unless stated otherwise. We want to define a functor "rig" from the category of formal $R$-schemes to the category of rigid $K$-spaces, which associates to a formal $R$-scheme $X$ its so-called 
generic fibre $X_{\text {rig. }}$ On affine formal $R$-schemes $\operatorname{Spf} A$ this functor is defined by

$$
\text { rig: } X=\operatorname{Spf} A \longmapsto X_{\text {rig }}=\operatorname{Sp}\left(A \otimes_{R} K\right),
$$

where we have seen at the beginning of this section that $A \otimes_{R} K$ is, indeed, an affinoid $K$-algebra and, hence, $\operatorname{Sp}\left(A \otimes_{R} K\right)$ an affinoid $K$-space. For any $f \in A$ we have

$$
\begin{aligned}
A\left\langle f^{-1}\right\rangle \otimes_{R} K & =[A\langle\zeta\rangle /(1-f \zeta)] \otimes_{R} K \\
& =\left(A \otimes_{R} K\right)\langle\zeta\rangle /(1-f \zeta)=\left(A \otimes_{R} K\right)\left\langle f^{-1}\right\rangle,
\end{aligned}
$$

showing that the functor rig produces from a basic open subspace of type

$$
X\left(f^{-1}\right)=\operatorname{Spf} A\left\langle f^{-1}\right\rangle \subset X=\operatorname{Spf} A
$$

the Laurent domain

$$
X_{\text {rig }}\left(f^{-1}\right)=\operatorname{Sp}\left(A \otimes_{R} K\right)\left\langle f^{-1}\right\rangle \subset X_{\text {rig }}=\operatorname{Sp}\left(A \otimes_{R} K\right)
$$

of the generic fibre associated to $X$. More generally, it follows that rig maps any open immersion of affine formal $R$-schemes $\operatorname{Spf} A^{\prime} \longrightarrow \operatorname{Spf} A$ to an open immersion $\operatorname{Sp}\left(A^{\prime} \otimes_{R} K\right) \longrightarrow \operatorname{Sp}\left(A \otimes_{R} K\right)$ between the associated affinoid $K$-spaces.

To extend the functor rig to global formal $R$-schemes, let us look at such a scheme $X$ and assume first that $X$ is separated and, hence, that the intersection of two open affine formal subschemes of $X$ is affine again. Thus, fixing an open affine covering $\left(U_{j}\right)_{j \in J}$ of $X$, all intersections $U_{j} \cap U_{j^{\prime}}$ are affine. Hence, we can glue the generic fibres $U_{j, \text { rig }}$ via the "intersections" $\left(U_{j} \cap U_{j^{\prime}}\right)_{\text {rig }}$ to produce a global rigid $K$-space $X_{\text {rig. }}$. It is easily checked that the latter is independent of the chosen affine open covering $\left(U_{j}\right)_{j \in J}$ of $X$ and that any morphism of separated formal $R$-schemes $X \longrightarrow Y$ leads to a canonical morphism $X_{\text {rig }} \longrightarrow Y_{\text {rig }}$ so that we really get a functor. In particular, as affine formal $R$-schemes are separated, the functor rig is defined on all open formal subschemes $U$ of an affine formal $R$-scheme $X$. Furthermore, since such a $U$ is necessarily quasi-compact, the generic fibre $U_{\text {rig }}$ is admissible open and, thus, an open subspace of $X_{\text {rig }}$. Therefore, to extend the functor rig to the category of all formal $R$-schemes, we can repeat the above construction, now interpreting an arbitrary global formal $R$-scheme $X$ by glueing open affine parts $U_{j}$ via arbitrary open subspaces of these. Thus, we have shown:

Proposition 5.4. The functor $A \longmapsto A \otimes_{R} K$ on R-algebras $A$ of topologically finite type gives rise to a functor rig: $X \longmapsto X_{\text {rig }}$ from the category of formal 
$R$-schemes, which are locally of topologically finite type, to the category of rigid $K$-spaces. We call $X_{\text {rig }}$ the generic fibre of $X$.

\section{Raynaud's approach to rigid geometry}

In view of 5.4, one would like to characterize all formal $R$-schemes $X$ whose generic fibre $X_{\text {rig }}$ coincides with a given rigid $K$-space $X_{K}$. To solve this problem, observe first that the functor $X \longmapsto X_{\text {rig }}$ factors through the category of admissible formal $R$-schemes, since the tensor product with $K$ over $R$ kills any $R$-torsion. Hence, the generic fiber of a given formal $R$-scheme $X$ coincides with the one of the induced admissible formal $R$-scheme obtained by killing $R$-torsion. Thus, we are reduced to the problem of describing all admissible formal $R$-schemes $X$ admitting a given rigid $K$-space $X_{\text {rig }}$ as generic fibre. To access this problem, we introduce the notion of admissible formal blowing-up.

Let $A$ be an $R$-algebra of topologically finite type and $M$ a finite $A$-module. Similarly as we have defined the structure sheaf $\mathcal{O}_{X}$ on the formal $R$-scheme Spf $A$, we may introduce the $\mathcal{O}_{X}$-module $M^{\Delta}$ associated to $M$. It is the sheaf extending the functor, which on basic open sets $D(f) \subset X$ for $f \in A$ is given by

$$
D(f) \longmapsto \lim _{n \in \mathbb{N}} M \otimes_{A} A /\left(I^{n}\right)\left[f^{-1}\right]=M \otimes_{A} A\left\langle f^{-1}\right\rangle
$$

see $[\mathrm{B}], 2.5 / 1$. As before, $I$ is a proper non-zero ideal in $R$. For any formal $R$-scheme $X$, an $\mathcal{O}_{X}$-module $\mathcal{F}$ is called coherent if there is an open affine covering $\left(X_{j}\right)_{j \in J}$ of $X$ such that the restriction $\left.\mathcal{F}\right|_{X_{j}}$ is associated to a finite $\mathcal{O}_{X_{j}}\left(X_{j}\right)$-module for all $j \in J$. One can show that this definition of coherence is independent of the chosen affine covering $\left(X_{j}\right)_{j \in J}$ of $X$ and that it is in accordance with the general concept which is customary for defining coherent sheaves; see [B], 2.5. To make this plausible, let us point out that any $R$-algebra of topologically finite presentation $A$ is a coherent ring in the sense that any finitely generated ideal of $A$ is of finite presentation; see [B], 2.3/6.

Definition 6.1. Let $X$ be a formal $R$-scheme which is locally of topologically finite presentation, and let $\mathcal{A} \subset \mathcal{O}_{X}$ be a coherent ideal which is open in the sense that, 
locally on $X$, it contains powers of tye $I^{n} \mathcal{O}_{X}$. Then the formal $R$-scheme

$$
X_{\mathcal{A}}=\lim _{n \in \mathbb{N}} \operatorname{Proj}\left(\bigoplus_{d=0}^{\infty} \mathcal{A}^{d} \otimes_{\mathcal{O}_{X}}\left(\mathcal{O}_{X} / I^{n} \mathcal{O}_{X}\right)\right)
$$

together with the canonical projection $X_{\mathcal{A}} \longrightarrow X$ is called the formal blowing-up of $\mathcal{A}$ on $X$. Any such blowing-up is referred to as an admissble formal blowing-up of $X$.

To look at admissible formal blowing-ups in more detail, let us consider an affine formal $R$-scheme $X=\operatorname{Spf} A$, where $A$ is an $R$-algebra of topologically finite presentation. Then it is more or less obvious that for any finitely generated open ideal $\mathfrak{a} \subset A$ and its associated coherent open ideal $\mathcal{A}=\mathfrak{a}^{\Delta}$ the formal blowing-up $X_{\mathcal{A}}$ of $\mathcal{A}$ on $X$ equals the $I$-adic completion of the scheme theoretic blowing-up ( $\operatorname{Spec} A)_{\mathfrak{a}}$ of $\mathfrak{a}$ on $\operatorname{Spec} A$; see [B], 2.6/6. Relying on this fact, one can derive quite precise information about the formal blowing-up $X_{\mathcal{A}}$, at least when $X$ is admissible; see [B], 2.6/7.

Proposition 6.2. Let $X=\operatorname{Spf} A$ be an admissible formal $R$-scheme which is affine, and let $\mathcal{A}=\mathfrak{a}^{\Delta}$ be a coherent open ideal in $\mathcal{O}_{X}$, which is associated to the coherent open ideal $\mathfrak{a}=\left(f_{0}, \ldots, f_{r}\right) \subset A$. Then the following assertions hold for the formal blowing-up $X_{\mathcal{A}}$ of $\mathcal{A}$ on $X$ :

(i) The ideal $\mathcal{A O}_{X_{\mathcal{A}}} \subset \mathcal{O}_{X_{\mathcal{A}}}$ is invertible; i. e., in terms of $\mathcal{O}_{X_{\mathcal{A}}}$ modules, it is locally isomorphic to $\mathcal{O}_{X_{\mathcal{A}}}$.

(ii) Let $U_{i}$ be the locus in $X_{\mathcal{A}}$, where $\mathcal{A O}_{X_{\mathcal{A}}}$ is generated by $f_{i}, i=0, \ldots, r$. Then the $U_{i}$ define an open affine covering of $X_{\mathcal{A}}$.

(iii) Write

$$
C_{i}=A\left\langle\frac{f_{j}}{f_{i}} ; j \neq i\right\rangle=A\left\langle\zeta_{j} ; j \neq i\right\rangle /\left(f_{i} \zeta_{j}-f_{j} ; j \neq i\right) .
$$

Then the I-torsion of $C_{i}$ coincides with its $f_{i}$-torsion, and we have $U_{i}=\operatorname{Spf} A_{i}$ with $A_{i}=C_{i} /(I$-torsion).

As a consequence we see that blowing up a coherent open ideal $\mathcal{A}$ on an admissible formal $R$-scheme yields an admissible formal $R$-scheme again. Furthermore, 
we can conclude that such blowing-ups are characterized by the universal property which is customary for blowing-up ([B], 2.6/9).

The explicit description of formal blowing-up in Proposition 6.2 is a key ingredient for understanding the relationship between formal $R$-schemes and their associated rigid $K$-spaces as generic fibres. To discuss this in more detail, we start with a basic observation.

Proposition 6.3. Let $X$ be an admissible formal $R$-scheme and $\mathcal{A} \subset \mathcal{O}_{X}$ a coherent open ideal. Then the functor

$$
\text { rig: (Formal } R \text {-schemes }) \longrightarrow \text { (Rigid } K \text {-spaces), } \quad \text { rig: } X \longmapsto X_{\text {rig }} \text {, }
$$

as introduced in Section 5, transforms the formal blowing-up $X_{\mathcal{A}} \longrightarrow X$ of $\mathcal{A}$ on $X$ into an isomorphism $\left(X_{\mathcal{A}}\right)_{\text {rig }} \stackrel{\sim}{\longrightarrow} X_{\text {rig }}$ between associated generic fibres.

In particular, the functor rig induces a functor

$$
\text { rig }^{\prime} \text { : (Formal } R \text {-schemes) formal blowing-up } \longrightarrow \text { (Rigid } K \text {-spaces) }
$$

from the category of admissible formal R-schemes, localized by admissible formal blowing-up, to the category of rigid $K$-spaces.

As it is quite instructive, we include the argument of proof. Certainly, the problem is local on $X$. Thus, we may assume that $X$ is affine, say $X=\operatorname{Spf} A$. Let the coherent open ideal $\mathcal{A} \subset \mathcal{O}_{X}$ be associated to the ideal $\mathfrak{a}=\left(f_{0}, \ldots, f_{r}\right)$ of $A$. Then it follows from Proposition 6.2 that $X_{\mathcal{A}}$ is covered by the affinoid $K$-spaces associated to the following admissible $R$-algebras:

$$
A_{i}=A\left\langle\frac{f_{0}}{f_{i}}, \ldots, \frac{f_{r}}{f_{i}}\right\rangle /(I \text {-torsion }), \quad i=0, \ldots, r
$$

Thus, applying the functor rig to the projection $\operatorname{Spf} A_{i} \longrightarrow \operatorname{Spf} A$ and writing $A_{K}=A \otimes_{R} K$, we obtain the canonical map

$$
\operatorname{Sp} A_{K}\left\langle\frac{f_{0}}{f_{i}}, \ldots, \frac{f_{r}}{f_{i}}\right\rangle \longrightarrow \operatorname{Sp} A_{K}
$$

which defines $X_{\text {rig }}\left(\frac{f_{0}}{f_{i}}, \ldots, \frac{f_{r}}{f_{i}}\right)$ as a rational subdomain of $X_{\text {rig }}=\operatorname{Sp} A_{K}$. More specifically, one checks that rig transforms the covering $\left(\operatorname{Spf} A_{i}\right)_{i=0 \ldots r}$ of $X_{\mathcal{A}}$ into 
the rational covering

$$
\bigcup_{i=0}^{n} X_{\text {rig }}\left(\frac{f_{0}}{f_{i}}, \ldots, \frac{f_{r}}{f_{i}}\right)=X_{\text {rig }}
$$

respecting intersections. Of course, one has to realize that, $\mathfrak{a}$ being open in $A$, it contains a power of $I$ so that the functions $f_{0}, \ldots, f_{r}$ will generate the unit ideal in $A_{K}$. This implies that rig transforms the morphism $X_{\mathcal{A}} \longrightarrow X$ into an isomorphism.

Under certain mild conditions we can strengthen Proposition 6.3 and show that the functor rig' is, in fact, an equivalence of categories. To give a precise statement, recall that a formal $R$-scheme $X$ is called quasi-paracompact if it admits an open covering by quasi-compact open subschemes $U_{j} \subset X, j \in J$, that is of finite type; i. e., such that each $U_{j}$ is disjoint from almost all other $U_{j^{\prime}}, j^{\prime} \in J$. Similarly, dealing with admissible coverings of finite type, the notion of quasi-paracompactness is defined for rigid $K$-spaces. Also note that a formal $R$-scheme $X$ is called separated if the diagonal embedding $\Delta: X \longrightarrow X \times X$ is a closed immersion, and quasi-separated if $\Delta$ is quasi-compact (which in our case, for $R$ a complete valuation ring, is automatic). Separatedness und quasiseparatedness are defined in the same way for rigid $K$-spaces.

Now we can state Raynaud's fundamental result [R1], [B], 2.8/3, which expresses the essence of his point of view on rigid geometry.

Theorem 6.4 (Raynaud). The functor

$$
\text { rig: (Formal } R \text {-schemes }) \longrightarrow \text { (Rigid } K \text {-spaces), } \quad \text { rig: } X \longmapsto X_{\text {rig }},
$$

induces via the functor rig' of Proposition 6.3 an equivalence between

(i) $(\mathrm{FSch} / R)_{S}$, which is the category of all quasi-paracompact admissible formal $R$-schemes, localized by the class $S$ of admissible formal blowing-ups, and

(ii) $(\mathrm{Rig} / K)$, which is the category of all quasi-separated quasi-paracompact rigid $K$-spaces.

For simplicity we have presented the theory of admissible $R$-algebras over complete valuation rings $R$ of hight 1 . But the theory works the same way over more general (complete and separated) adic rings with a finitely generated ideal of 
definition $I \subset R$ such that $R$ does not have $I$-torsion. Of particular interest are the following types of rings:

(V) $R$ is an adic valuation ring with a finitely generated ideal of definition I (which automatically is principal then).

(N) $R$ is a noetherian adic ring with an ideal of definition $I$ where $R$ does not have I-torsion.

Instead of $S=\operatorname{Spf} R$ we can just as well work over more global bases. The following types of formal base schemes $S$ will be of interest:

$\left(\mathrm{V}^{\prime}\right) S$ is an admissible formal $R$-scheme, where $R$ is an adic valuation ring of type $(\mathrm{V})$ as above. Thus, the topology of $\mathcal{O}_{S}$ is generated by the ideal $I \mathcal{O}_{S}$.

$\left(\mathrm{N}^{\prime}\right) S$ is a noetherian formal scheme (of quite general type) such that the topology of its structure sheaf $\mathcal{O}_{S}$ is generated by a coherent ideal $\mathcal{I} \subset \mathcal{O}_{S}$ and such that $\mathcal{O}_{S}$ does not admit $\mathcal{I}$-torsion.

For base schemes $S$ of this type, it is possible to consider admissible formal $S$-schemes, or just formal $S$-schemes, which are locally of topologically finite presentation. Then Raynaud's Theorem 6.4 enables us to extend the notion of rigid spaces to such more general situations:

Definition 6.5. Let $S$ be a formal scheme of type $\left(\mathrm{V}^{\prime}\right)$ or $\left(\mathrm{N}^{\prime}\right)$, as defined above, and let $(\mathrm{FSch} / S)$ be the category of admissible formal $S$-schemes. Then the category $(\mathrm{Rig} / S)$ of rigid $S$-spaces is defined as the localization of $(\mathrm{FSch} / S)$ by admissible formal blowing-ups.

Thus, as object, a rigid $S$-space is the same as an admissible formal $S$-scheme, whereas on the level of morphisms, admissible formal blowing-ups are viewed as isomorphisms. Any admissible formal $S$-scheme representing a rigid $S$-space $X_{\text {rig }}$ will be called a formal model of $X_{\text {rig }}$. 


\section{Some results on formal models}

Let $S$ be a formal base scheme of type $\left(\mathrm{V}^{\prime}\right)$ or $\left(\mathrm{N}^{\prime}\right)$, as considered above, and let $X_{\text {rig }}$ be a rigid $S$-space in the sense of 6.5 . Without explicitly saying so, we will always assume such rigid spaces, as well as their formal $S$-models, to be quasi-separated and quasi-paracompact.

If $(P)$ is a property applicable to schemes or formal schemes, we can basically proceed in two ways in order to extend the notion of $(P)$ to rigid $S$-spaces like $X_{\text {rig. }}$. The first possibility is to say that $X_{\text {rig }}$ satisfies $(P)$ if there is a formal $S$-model $X$ of $X_{\text {rig }}$ satisfying $(P)$. For example, on the level of morphisms, one can proceed like this with open (resp. closed) immersions. Thus, call a morphism of rigid $S$-spaces $\tau_{\text {rig }}: U_{\text {rig }} \longrightarrow X_{\text {rig }}$ an open immersion (resp. a closed immersion) if $\tau_{\text {rig }}$ admits an open (resp. closed) immersion of admissible formal $S$-schemes $\tau: U \longrightarrow X$ as a formal $S$-model. That such a definition coincides with the usual one in the classical rigid case, follows from [B], 2.8/4.

Another interesting property $(P)$ to look at is the notion of properness. The latter has been introduced by Kiehl [K2] for morphisms of classical rigid spaces $\varphi_{K}: X_{K} \longrightarrow Y_{K}$ over some field $K$. If $\varphi: X \longrightarrow Y$ is a formal model of $\varphi_{K}$, it is not hard to see that $\varphi$ will be proper in the sense that the associated morphism between special fibers

$$
\varphi \otimes_{R} R / I: X \otimes_{R} R / I \longrightarrow Y \otimes_{R} R / I
$$

is a proper morphism of schemes. That the converse is also true, has been an open question for quite a long time. It was finally proved by Lütkebohmert [L2] over discrete valuation rings $R$ and by Temkin [Te2] in the general case. Switching from proper morphisms of rigid spaces to proper formal models opens up a convenient way to reprove basic theorems of classical rigid geometry, like the Proper Mapping Theorem [K2] and related results on Serre's GAGA-functor [S]; see [L2], [U].

Returning to the general case, a more direct possibility of defining a property $(P)$ on rigid spaces is to look at the validity of $(P)$ on the "complement" of the special fibre of formal $S$-models $X$ associated to $X_{\text {rig. }}$. To be more precise, let $\mathcal{I} \subset \mathcal{O}_{S}$ be an ideal of definition. Then, for any formal $S$-model $X$, the scheme $X_{0}=X \otimes_{S} \mathcal{O}_{S} / \mathcal{I}$ is called the special fibre of $X$. If $\left(U_{i}\right)_{i \in J}$ is an affine open covering of $X$, say $U_{i}=\operatorname{Spf} A_{i}$, and if, on $U_{i}$, the coherent open ideal $\mathcal{I} \mathcal{O}_{X} \subset \mathcal{O}_{X}$ is associated to the ideal $\mathfrak{a}_{i} \subset A_{i}$, we view the ordinary scheme $\operatorname{Spec} A_{i}-V\left(\mathfrak{a}_{i}\right)$, 
locally on $U_{i}$, as the complement of the special fibre, although such a complement is not well-defined globally. Now, if $(P)$ is a scheme property, we can say that $X_{\text {rig }}$ satisfies $(P)$ if all schemes $\operatorname{Spec} A_{i}-V\left(\mathfrak{a}_{i}\right)$ satisfy $(P)$. Of course, in order that $(P)$ defines a reasonable property on the associated rigid $S$-space $X_{\text {rig }}$, one has to check that the validity of $(P)$ is independent of the chosen covering $\left(U_{i}\right)_{i \in J}$ of $X$ and invariant under admissible formal blowing-up. Then, in general, it is a truely demanding venture, to find out, whether or not a rigid $S$-space satisfying $(P)$ will always admit a formal $S$-model satisfying $(P)$.

As a first example which can successfully be handled along these lines, let us mention the property $(P)$ of being flat, for morphisms of rigid $S$-spaces or coherent modules on rigid $S$-spaces. This notion of flatness is compatible with the usual one, known for classical rigid spaces. The main result on flatness is the existence of flat formal models, due to Raynaud and Gruson; see [RG] or [FRG2].

Theorem 7.1. Let $\varphi: X \longrightarrow Y$ be a quasi-compact morphism of admissible formal $S$-schemes, and assume that the associated morphism of rigid $S$-spaces $\varphi_{\text {rig }}: X_{\text {rig }} \longrightarrow Y_{\text {rig }}$ is flat. Then there exists a commutative diagram of admissible formal S-schemes

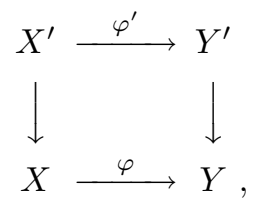

where $\varphi^{\prime}$ is flat, $Y^{\prime} \longrightarrow Y$ is the formal blowing-up of some coherent open ideal $\mathcal{A} \subset \mathcal{O}_{Y}$, and where $X^{\prime} \longrightarrow X$ is the formal blowing-up of the ideal $\mathcal{A O}_{X} \subset \mathcal{O}_{X}$ on $X$.

Let us mention that $X^{\prime}$ can also be viewed as the strict transform of $X$ with respect to the admissible formal blowing-up $Y^{\prime} \longrightarrow Y$. The latter is constructed from the fibered product $X^{\prime \prime}=X \times_{Y} Y^{\prime}$ (a formal $S$-scheme of locally topologically finite presentation, but not necessarily admissible) by dividing the structure sheaf by all torsion with respect to the ideal generated by the pull-back of $\mathcal{A}$. The existence of flat models has an interesting consequence for classical rigid spaces.

Corollary 7.2. In the classical rigid case, let $R$ be an adic valuation ring of height 1 with field of fractions $K$. Furthermore, let $\varphi_{K}: X_{K} \longrightarrow Y_{K}$ be a flat 
morphism of quasi-compact and quasi-separated rigid $K$-spaces. Then its image $\varphi_{K}\left(X_{K}\right)$ is admissible open in $Y_{K}$.

Proof. Due to Theorem 7.1, there exists a flat formal $R$-model $\varphi: X \longrightarrow Y$ of $\varphi_{K}$. Tensoring $\varphi$ with the residue field $k$ of $R$ yields a morphism of $k$-schemes $\varphi_{k}: X_{k} \longrightarrow Y_{k}$, which is flat and of finite presentation. It is known that the image of $\varphi_{k}$ is a quasi-compact open subscheme $V_{k} \subset Y_{k}$; see [EGA], 2.4.6. Now, if $V \subset Y$ is the corresponding open formal subscheme of $Y$, then, clearly, $\varphi$ factors through $V$, and the induced morphism $X \longrightarrow V$ is faithfully flat. Finally, a local consideration involving rig-points, as considered in [B], 2.7, shows that $\varphi_{K}$ must map $X_{K}$ onto the admissible open subspace $V_{\text {rig }} \subset X_{K}$, which is associated to $V$.

Another property $(P)$, which can be defined on general rigid $S$-spaces $X_{\text {rig }}$ by requiring $(P)$ to be satisfied on the "complement" of the special fibre, is the notion of smoothness. Also in this case, one may ask if any smooth (or even étale) morphism of rigid $S$-spaces will admit a smooth (resp. étale) formal $S$-model. However, the answer will be negative in general. Thus, expecting the existence of smooth formal $S$-models of smooth rigid $S$-spaces would be too much. Stepping back a bit, one may replace smoothness by the weaker property $(P)$ that the structural morphism $X_{\text {rig }} \longrightarrow S_{\text {rig }}$ has geometrically reduced fibres. Here is an advanced result on the existence of formal $S$-models with such a property $(P)$ :

Reduced Fiber Theorem 7.3. Let $X$ be a quasi-compact admissible formal $S$-scheme such that $X / S$ is flat and $X_{\text {rig }} / S_{\text {rig }}$ has reduced geometric fibres, equidimensional of dimension $d$. Then there is a commutative diagram of admissible formal S-schemes

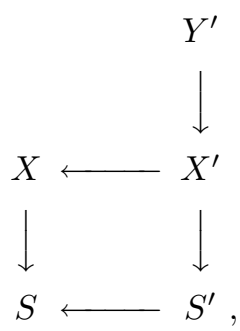

The theorem has been proved in [FRG4] in the classical rigid case and in the noetherian case $\left(\mathrm{N}^{\prime}\right)$. 
where

(i) $X^{\prime}=X \times_{S} S^{\prime}$,

(ii) $S^{\prime} \longrightarrow S$ is surjective and $S_{\text {rig }}^{\prime} \longrightarrow S_{\text {rig }}$ is étale,

(iii) $Y^{\prime} \longrightarrow X^{\prime}$ is finite and $Y_{\text {rig }}^{\prime} \longrightarrow X_{\text {rig }}^{\prime}$ is an isomorphism,

(iv) $Y^{\prime} \longrightarrow S^{\prime}$ is flat and has reduced geometric fibres.

Note that, due to Theorem 7.1, the assumption of $X / S$ to be flat may be replaced by requiring $X_{\text {rig }} / S_{\text {rig }}$ to be flat. Furthermore, at least in the noetherian case $\left(\mathrm{N}^{\prime}\right)$, the assumption on the euqidimensionality of the fibres of $X_{\text {rig }} / S_{\text {rig }}$ can be avoided. Let us also mention that the Reduced Fiber Theorem may be viewed as a relative version of a Finiteness Theorem of Grauert and Remmert [GR].

\section{References}

[A] M. Artin: Grothendieck topologies. Notes on a seminar by M. Artin, Harvard University (1962)

[Ba] W. Bartenwerfer: Der Kontinuitätssatz für rein-dimensionale $k$-affinoide Räume. Math. Ann. 193, 139-170 (1971)

[B1] V. Berkovich: Spectral theory and analytic geometry over non-Archimedean fields. Mathematical Surveys and Monographs, Vol. 33, American Mathematical Society (1990)

[B2] V. Berkovich: Etale cohomology for non-Archimedean analytic spaces. Publ. Math. IHES 78 (1993)

[Bp] A. Bertapelle: Formal Néron models and Weil restriction. Math. Ann. 316, 437-463 (2000)

[B] S. Bosch: Lectures on formal and rigid geometry. Preprint 378 of the SFB Geometrische Strukturen in der Mathematik, Münster (2005), http://wwwmath1.uni-muenster.de/sfb/about/publ/heft378.ps

[BGR] S. Bosch, U. Güntzer, R. Remmert: Non-Archimedean Analysis. Grundlehren der Mathematischen Wissenschaften Vol. 261, Springer (1984)

[BL1] S. Bosch, W. Lütkebohmert: Stable reduction and uniformization of abelian varieties I. Math. Ann. 270, 349-379 (1985)

[BL2] S. Bosch, W. Lütkebohmert: Stable reduction and uniformization of abelian varieties II. Invent. Math. 78, 257-297 (1984)

[BL3] S. Bosch, W. Lütkebohmert: Degenerating abelian varieties. Topology 30, 653-698 (1991)

[FRG1] S. Bosch, W. Lütkebohmert: Formal and rigid geometry, I. Rigid spaces. Math. Ann. 295, 291-317 (1993) 
[FRG2] S. Bosch, W. Lütkebohmert: Formal and rigid geometry, II. Flattening techniques. Math. Ann. 296, 403-429 (1993)

[FRG3] S. Bosch, W. Lütkebohmert, M. Raynaud: Formal and rigid geometry, III. The relative maximum principle. Math. Ann. 302, 1-29 (1995)

[FRG4] S. Bosch, W. Lütkebohmert, M. Raynaud: Formal and rigid geometry, IV. The reduced fibre theorem. Invent. Math. 119, 361-398 (1995)

[EGA] A. Grothendieck, J. Dieudonné: Eléments de Géométrie Algébrique $\mathrm{IV}_{2}$. Publ. Math. IHES 24 (1964)

[F] K. Fujiwara: Theory of tubular neighborhood in étale topology. Duke Math. J. 80, 15-57 (1995)

[FC] G. Faltings, C.-L. Chai: Degeneration of abelian varieties. Ergebnisse der Mathematik und ihrer Grenzgebiete, 3. Folge, Bd. 22. Springer (1990)

[GG] L. Gerritzen, H. Grauert: Die Azyklizität der affinoiden Überdeckungen. Global Analysis, Papers in Honor of K. Kodaira, 159-184. University of Tokyo Press, Princeton University Press (1969)

[GR] H. Grauert, R. Remmert: Über die Methode der diskret bewerteten Ringe in der nichtarchimedischen Analysis. Invent. Math. 2, 87-133 (1966)

$[\mathrm{H}] \quad$ R. Huber: Etale cohomology of rigid analytic varieties and adic spaces. Aspects of Mathematics, Vol. E 30, Vieweg (1996)

[K1] R. Kiehl: Theorem A und Theorem B in der nichtarchimedischen Funktionentheorie. Invent. Math. 2, 256-273, (1967)

[K2] R. Kiehl: Der Endlichkeitssatz für eigentliche Abbildungen in der nichtarchimedischen Funktionentheorie. Invent. Math. 2, 191-214, (1967)

[Kö] U. Köpf: Über eigentliche Familien algebraischer Varietäten über affinoiden Räumen. Schriftenr. Math. Inst. Univ. Münster, Heft 7 (1974)

[L1] W. Lütkebohmert: Fortsetzbarkeit $k$-meromorpher Funktionen. Math. Ann. 220, 273284 (1976)

[L2] W. Lütkebohmert: Formal-algebraic and rigid-analytic geometry. Math. Ann. 286, 341371 (1990)

[M1] D. Mumford: An analytic construction of curves with degenerate reduction over complete local rings. Comp. Math. 24, 129-174 (1974)

[M2] D. Mumford: An analytic construction of degenerating abelian varieties over complete rings. Comp. Math. 24, 239-272 (1972)

[R1] M. Raynaud: Géométrie analytique rigide d'apres Tate, Kiehl, ... Table ronde d'analyse non archimedienne. Bull. Soc. Math. France, Mémoire 39/40, 319-327 (1974)

[R2] M. Raynaud: Variétés abéliennes et géométrie rigide. Actes du congrès international des Mathématiciens (Nice 1970), tome 1, 473-477 (1971)

[RG] M. Raynaud, L. Gruson: Critères de platitude et de projectivité. Invent. Math. 13, 1-89 (1971)

[S] J.-P. Serre: Géométrie algébrique et géométrie analytique. Ann. Inst. Fourier 6, 1-42 (1956) 
[T] J. Tate: Rigid analytic spaces. Private notes, reproduced with(out) his permission by I. H. E. S. (1962). Reprinted in Invent. Math. 12, 257-289 (1971)

[Te1] M. Temkin: A new proof of the Gerritzen-Grauert theorem. Math. Ann. 333, 261-269 (2005)

[Te2] M. Temkin: On local properties of non-Archimedean analytic spaces. Math. Ann. 318, 585-607 (2000)

[U] P. Ullrich: The direct image theorem in formal and rigid geometry. Math. Ann. 301, 69-104 (1995)

Siegfried Bosch

Mathematisches Institut der Universität

Einsteinstr. 62, 48149 Münster, Germany

E-mail: bosch@math.uni-muenster.de 